Int. J. Contemp. Math. Sciences, Vol. 2, 2007, no. 6, $261-273$

\title{
A Basic Canonical Form of Discrete-Time Compartmental Systems
}

\author{
Rafael Bru, Rafael Cantó, Beatriz Ricarte \\ Institut de Matemàtica Multidisciplinar. \\ Universitat Politècnica de València. \\ Camí de Vera s/n, 46022 València, Spain. \\ \{rbru,rcanto,bearibe\}@mat.upv.es \\ Ventsi Rumchev \\ Dept. of Mathematics and Statistics. \\ Curtin University of Technology. \\ P.O. Box U 1987, Perth, WA 6845, Australia. \\ rumchevv@maths.curtin.edu.au
}

\begin{abstract}
The transfer functions of discrete-time SISO compartmental systems whose compartments are connected either in series or in parallel are characterized, and then the corresponding realization problem is studied. The obtained results are extended to the transfer matrix of $\mathrm{N}$ periodic compartmental systems.
\end{abstract}

Mathematics Subject Classification: 93B10, 93B15, 93B20, 93C55

Keywords: Compartmental systems, discrete-time, transfer matrix, minimal realization

\section{Introduction}

It is well known that a compartmental system consists of a finite number of interconnected subsystems called compartments. The interaction among compartments are transfers of material according to the law of conservation of mass. These are natural models useful for many areas of application subject to that law appearing in physiology, chemistry, medicine, epidemiology, ecology, pharmacokinetics, and economy $[2,5,8,9,14]$. The state variables of these systems represent the amount of material contained in each compartment and 
then they are restricted to being nonnegative over time; that is, they belong to the broader class of positive systems.

Compartmental systems, as any control system, can be represented either in the space-state model by a system of equations where the vector states are unknown or in the input-output model where the output vector is determined by a transfer matrix function. Is it possible to identify the inner structure of a system, that is to say, a space-state model starting from its input-output model? This is the realization problem which is solved for any general control system; however, it remains unsolved when we restrict the problem to systems with constraints such as positive systems and, in particular, compartmental systems.

The problem of positive realizations in continuous-time has been studied by different authors $[1,3,8,12]$, yet those in discrete-time have received less attention. Concretely, Kaczorek [10] analyze positive realization problems for linear continuous-time and discrete-time systems using positive canonical forms. Ohta, Maeda and Kodama [13] dealt with these questions by means of the theory of convex cone, and Bru, Coll, Hernández and Sánchez [6, 7] extended these results to periodic positive systems.

The aim of this paper is to characterize the transfer matrix of discrete-time SISO compartmental systems with compartments connected in series or in parallel and then to analyze the corresponding realization problem. We prove that these transfer functions admit a minimal asymptotically stable or simply stable compartmental canonical form which we have called a basic bidiagonal realization. Afterwards, we examine the case of $N$-periodic compartmental systems knowing that any $N$-periodic system can be represented by $N$ associated invariant systems.

\section{Compartmental Realizations}

A control system with $n$ states can be represented, in the space-state model, by the system

$$
\left.\begin{array}{ll}
x(k+1) & =A(k) x(k)+B(k) u(k) \\
y(k) & =C(k) x(k), \quad k \in \mathbb{Z}
\end{array}\right\}
$$

where $A(k) \in \mathbb{R}^{n \times n}, B(k) \in \mathbb{R}^{n \times m}$ and $C(k) \in \mathbb{R}^{r \times n}$. When all matrices are constant, the system is invariant and is denoted by $(A, B, C)$.

The invariant systems can also be represented by an input-output model relating inputs and outputs by a rational matrix of size $r \times m$ called the transfer matrix and is denoted by $G(z)$. Particularly in discrete-time, a transfer matrix relates the $\mathcal{Z}$ transformations of outputs and inputs, i.e.,

$$
\hat{y}(z)=G(z) \hat{u}(z)
$$


Every matrix $G(z)$ is associated with infinite space-state models, each of these representations is called a realization of $G(z)$. More precisely, given a strictly proper matrix $G(z) \in \mathbb{R}^{r \times m}(z)$, the triple $(A, B, C), A \in \mathbb{R}^{n \times n}$, $B \in \mathbb{R}^{n \times m}, C \in \mathbb{R}^{r \times n}$ that satisfies

$$
G(z)=C(z I-A)^{-1} B
$$

is said to be a realization of $G(z)$. The size of $A$ is called the dimension of the realization. Moreover, this realization will be positive if $A, B$ and $C$ are nonnegative; compartmental if it is a positive realization and the entries of $A$ satisfy that $\sum_{i=1}^{n} a_{i j} \leq 1$, for all $j=1,2, \ldots, n$. In this case, the matrix $A$ is called a compartmental matrix; minimal if it has a minimum dimension and (lower) bidiagonal if $A$ has only two nonzero diagonals, the main diagonal and the immediate lower diagonal.

The transfer function of a SISO system can be mathematically split in transfer functions of a smaller order, which is equivalent to splitting the system in interconnected simpler subsystems, as restated below. We recall that two subsystems are connected in series if the output of the first subsystem is the input of the second subsystem. The transfer function of the overall system $G(z)$ is obtained by multiplying the two transfer functions $G_{1}(z)$ and $G_{2}(z)$ of these two subsystems. Otherwise, two subsystems are connected in parallel if they have the same input and the output of the overall system is the sum of their outputs. Likewise, the transfer function of the overall system $G(z)$ is the sum of their transfer functions.

Definition 2.1 The transfer function of a simple compartmental discrete-time linear system is given by

$$
C(z)=\frac{\alpha}{z-a}, \quad \alpha>0, \quad 0 \leq a \leq 1
$$

Remark 2.2 Note that the transfer function

$$
G(z)=\sum_{i=1}^{n} \frac{k_{i}}{\prod_{r=i}^{n}\left(z-a_{r}\right)}
$$

with $k_{i}>0$ and $0 \leq a_{r} \leq 1, i, r=1,2, \ldots, n$, represents a SISO compartmental system with $n$ compartments connected in series where the input $u(z)$ has influences on every compartment but only the last one affects the output $y(z)$.

\section{Basic Bidiagonal Realizations}

The realization problem of linear systems normally uses classical canonical forms as the reachable and controllable forms or diagonal and Jordan forms. 
But often these forms are not useful in positive systems and so nor in compartmental systems, because those canonical forms are not positive realizations. Now, we define another basic form that is an attractive alternative to conventional canonical forms for SISO positive and compartmental systems.

Definition 3.1 A realization $(A, B, C)$ of a discrete-time invariant system is called a basic bidiagonal realization if

$$
A=\left[\begin{array}{cccc}
a_{11} & & & \\
a_{21} & \ddots & & \\
& \ddots & \ddots & \\
& & a_{n, n-1} & a_{n n}
\end{array}\right], B=\left[\begin{array}{c}
b_{1} \\
b_{2} \\
\vdots \\
b_{n}
\end{array}\right], C=\left[\begin{array}{lll}
c_{1} & c_{2} \cdots & c_{n}
\end{array}\right]
$$

with the entries $a_{i i} \geq 0, a_{i, i+1} \geq 0, a_{i j}=0$ for $j \neq i, i+1, \ldots \quad b_{j} \geq 0$ and $c_{i} \geq 0, i, j=1,2, \ldots, n$, and where the columns of $A$ satisfy $\sum_{i=1}^{n} a_{i j} \leq 1$, for all $j=1,2, \ldots, n$.

Note that this realization is a compartmental representation of the given system. It can only represent systems whose transfer functions have nonnegative real poles, because the eigenvalues of the matrix $A$ must be nonnegative real numbers due to its lower triangular form. In addition, these real poles are less than or equal to 1 given its compartmental condition. Moreover, in this case, when the system is simply stable, some $a_{i i}=1$ and the corresponding column entry must be zero.

We recall that a discrete-time system $(A, B, C)$ is (i) asymptotically stable if and only if the spectral radius $\rho(A)<1$; (ii) the system is simply stable (or marginally stable) if $\rho(A)=1$ and all eigenvalues $|\lambda|=1$ are simple roots of the minimal polynomial of $A$; and (iii) otherwise, the system is considered unstable.

The concept of diagonally, algebraically equivalent positive discrete-time systems for difference algebraic equations appears in [11] where the authors show that any two minimal realizations are diagonally algebraically equivalent (under some conditions) for systems without controls. The following result relates a positive bidiagonal realization with the basic bidiagonal realization in discrete-time systems.

Lemma 3.2 Any asymptotically stable positive bidiagonal realization is diagonally similar to an asymptotically stable basic bidiagonal realization.

Now, we examine the case when a transfer function $G(z)$ admits a basic bidiagonal realization. We begin with the case of the asymptotically stable transfer function. 
Theorem 3.3 Let $G(z)$ be a strictly proper rational asymptotically stable transfer function. Then the following conditions are equivalent:

a) $G(z)$ admits a minimal basic bidiagonal realization with $a_{i j}>0, b_{j}>0$ and $c_{i}>0, i, j=1,2, \ldots, n$, which is asymptotically stable.

b) $G(z)$ can be given as:

$$
G(z)=\sum_{j=1}^{n}\left(\sum_{i=j}^{n} \frac{k_{i j}}{\prod_{r=j}^{i}\left(z-a_{r}\right)}\right)
$$

where $0 \leq a_{r}<1, r=1,2, \ldots, n$ and $k_{i j}>0, i, j=1,2, \ldots, n, i \geq j$.

Proof.

a) $\Rightarrow \mathrm{b})$ Let $(A, B, C)$ be an asymptotically stable basic bidiagonal realization of $G(z)$ with $a_{i j}>0, b_{j}>0$ and $c_{i}>0, i, j=1,2, \ldots, n$, with the structure given in (1). The corresponding transfer function is given by

$$
\begin{aligned}
& G(z)=C(z I-A)^{-1} B=\frac{c_{1} b_{1}}{\left(z-a_{11}\right)}+\frac{c_{2} a_{21} b_{1}}{\left(z-a_{22}\right)\left(z-a_{11}\right)}+\cdots+\frac{c_{n} a_{21} a_{32} \cdots a_{n, n-1} b_{1}}{\left(z-a_{n n}\right) \cdots\left(z-a_{11}\right)}+ \\
& \quad+\frac{c_{2} b_{2}}{\left(z-a_{22}\right)}+\frac{c_{3} a_{32} b_{2}}{\left(z-a_{33}\right)\left(z-a_{22}\right)}+\cdots+\frac{c_{n} a_{32} a_{43} \cdots a_{n, n-1} b_{2}}{\left(z-a_{n n}\right) \cdots\left(z-a_{22}\right)}+ \\
& \quad+\cdots+\frac{c_{n-1} b_{n-1}}{\left(z-a_{n-1, n-1}\right)}+\frac{c_{n} a_{n, n-1} b_{n-1}}{\left(z-a_{n-1, n-1}\right)\left(z-a_{n n}\right)}+\frac{c_{n} b_{n}}{\left(z-a_{n n}\right)}
\end{aligned}
$$

and then, $G(z)$ has the structure given in (2). More precisely, comparing both expressions, we have

$$
a_{i}=a_{i i} \quad k_{i i}=c_{i} b_{i} \quad k_{i j}=c_{i} \prod_{s=j}^{i-1} a_{s+1, s} b_{j}
$$

with $i, j=1,2, \ldots, n, i \geq j$, and consequently,

$$
G(z)=\sum_{j=1}^{n}\left(\sum_{i=j}^{n} \frac{k_{i j}}{\prod_{r=j}^{i}\left(z-a_{r}\right)}\right)
$$

where $0 \leq a_{r}<1, r=1,2, \ldots, n$ and $k_{i j}>0, i, j=1,2, \ldots, n, i \geq j$. 
b) $\Rightarrow$ a) Let splitting the transfer function (2) as

$$
\begin{aligned}
G(z)= & \sum_{j=1}^{n}\left(\sum_{i=j}^{n} \frac{k_{i j}}{\prod_{r=j}^{i}\left(z-a_{r}\right)}\right)= \\
= & \frac{k_{11}}{\left(z-a_{1}\right)}+\frac{k_{21}}{\left(z-a_{2}\right)\left(z-a_{1}\right)}+\cdots+\frac{k_{n 1}}{\left(z-a_{n}\right) \cdots\left(z-a_{1}\right)}+ \\
& +\frac{k_{22}}{\left(z-a_{2}\right)}+\frac{k_{32}}{\left(z-a_{3}\right)\left(z-a_{2}\right)}+\cdots+\frac{k_{n 2}}{\left(z-a_{n}\right) \cdots\left(z-a_{2}\right)}+ \\
& +\cdots+\frac{k_{n-1, n-1}}{\left(z-a_{n-1}\right)}+\frac{k_{n, n-1}}{\left(z-a_{n}\right)\left(z-a_{n-1}\right)}+\frac{k_{n n}}{\left(z-a_{n}\right)}= \\
= & \frac{k_{11}}{\left(z-a_{1}\right)}+\left(\frac{k_{21}}{\left(z-a_{2}\right)\left(z-a_{1}\right)}+\frac{k_{22}}{\left(z-a_{2}\right)}\right)+ \\
& +\left(\frac{k_{31}}{\left(z-a_{3}\right)\left(z-a_{2}\right)\left(z-a_{1}\right)}+\frac{k_{32}}{\left(z-a_{3}\right)\left(z-a_{2}\right)}+\frac{k_{33}}{\left(z-a_{3}\right)}\right)+ \\
& +\cdots+\left(\sum_{i=1}^{n} \frac{k_{n i}}{\prod_{j=i}^{n}\left(z-a_{j}\right)}\right)= \\
= & G_{1}(z)+G_{2}(z)+G_{3}(z)+\cdots+G_{n}(z) .
\end{aligned}
$$

Therefore,

$$
\begin{aligned}
y(z) & =\left[G_{1}(z)+G_{2}(z)+G_{3}(z)+\cdots+G_{n}(z)\right] u(z)= \\
& =y_{1}(z)+y_{2}(z)+y_{3}(z)+\cdots+y_{n}(z)
\end{aligned}
$$

By Remark 2.2, each $G_{i}(z), i=1,2, \ldots, n$, represents a SISO compartmental subsystem with $n$ compartments connected in series where the input $u(z)$ influences all compartments, but only the last one has an output. Focusing on each subsystem, we find

$$
\begin{aligned}
y_{1}(z) & =\frac{\alpha_{1}}{\left(z-a_{1}\right)} \beta_{1} u(z)=x_{1}(z) \\
y_{2}(z) & =\frac{\alpha_{1}}{\left(z-a_{1}\right)} \frac{\alpha_{2}}{\left(z-a_{2}\right)} \beta_{1} u(z)+\frac{\alpha_{2}}{\left(z-a_{2}\right)} \beta_{2} u(z)= \\
& =\frac{\alpha_{2}}{\left(z-a_{2}\right)} x_{1}(z)+\frac{\alpha_{2}}{\left(z-a_{2}\right)} \beta_{2} u(z)=x_{2}(z) \\
& \vdots \\
y_{n}(z) & =\frac{\alpha_{1}}{\left(z-a_{1}\right)} \frac{\alpha_{2}}{\left(z-a_{2}\right)} \cdots \frac{\alpha_{n}}{\left(z-a_{n}\right)} \beta_{1} u(z)+\frac{\alpha_{2}}{\left(z-a_{2}\right)} \cdots \frac{\alpha_{n}}{\left(z-a_{n}\right)} \beta_{2} u(z)+\cdots+ \\
& +\frac{\alpha_{n}}{\left(z-a_{n}\right)} \beta_{n} u(z)=\frac{\alpha_{n}}{\left(z-a_{n}\right)} x_{n-1}(z)+\frac{\alpha_{n}}{\left(z-a_{n}\right)} \beta_{n} u(z)=x_{n}(z) .
\end{aligned}
$$


Since $k_{i j}>0$, for all $i$ and $j$, we take $\alpha_{i}>0, \beta_{i}>0$ and using the $\mathcal{Z}$ inverse transformation, we obtain the following system:

$$
\begin{aligned}
& x_{1}(k+1)=a_{1} x_{1}(k)+\alpha_{1} \beta_{1} u(k) \\
& x_{2}(k+1)=\alpha_{2} x_{1}(k)+a_{2} x_{2}(k)+\alpha_{2} \beta_{2} u(k) \\
& \begin{array}{c}
\quad \begin{array}{c}
\vdots \\
x_{n}(k+1)=
\end{array} \quad \alpha_{n} x_{n-1}(k)+a_{n} x_{n}(k)+\alpha_{n} \beta_{n} u(k)
\end{array} \\
& y(k)=x_{1}(k)+x_{2}(k)+x_{3}(k)+\cdots+x_{n}(k)
\end{aligned}
$$

which can be written as:

$$
\left.\begin{array}{ll}
x(k+1) & =A x(k)+B u(k) \\
y(k) & =C x(k)
\end{array}\right\}
$$

where

$$
A=\left[\begin{array}{ccccc}
a_{1} & & & & \\
\alpha_{2} & a_{2} & & & \\
& \alpha_{3} & a_{3} & & \\
& & \ddots & \ddots & \\
& & & \alpha_{n} & a_{n}
\end{array}\right], \quad B=\left[\begin{array}{c}
\alpha_{1} \beta_{1} \\
\alpha_{2} \beta_{2} \\
\alpha_{3} \beta_{3} \\
\vdots \\
\alpha_{n} \beta_{n}
\end{array}\right], \quad C=\left[\begin{array}{lllll}
1 & 1 & 1 & \cdots & 1
\end{array}\right] .
$$

Note that it is a positive realization since $\alpha_{i}>0$ and $\beta_{i}>0, i=1,2, \ldots, n$. Now, considering Lemma 3.2, we can obtain a compartmental realization which will be bidiagonal, asymptotically stable and minimal since the order of $A$ is $n$ where $n$ is the degree of the denominator of $G(z)$.

Corollary 3.4 Theorem 3.3 is also valid for $k_{i j} \geq 0$ but taking into account some restrictions, i.e., if a constant $k_{i_{0} j_{0}}=0$, then one of the following conditions hold:

i) $k_{i_{0} r}=0$ for all $r=1,2, \ldots, i_{0}$

ii) given an $s, j_{0} \leq s \leq i_{0}-1, k_{r t}=0$, for all $r=s+1, s+2, \ldots, n$ and $t=1,2, \ldots, s$

iii) $k_{r j_{0}}=0$ for all $r=j_{0}, j_{0}+1, \ldots, n$

Proof. The restrictions of $k_{i j}$ can be obtained by (3). In fact, if $k_{i_{0} j_{0}}=0$, at least one of the following three contitions holds:

i) $c_{i_{0}}=0$. Then all the constants in which this coefficient appears will be zero, that is, $k_{i_{0} r}=0$ for all $r=1,2, \ldots, i_{0}$; 
ii) $\prod_{s=j_{0}}^{i_{0}-1} a_{s+1, s}=0$. In this case, some coefficients $a_{s+1, s}$ are zero. Therefore, the constant $k_{r t}$ will also be zero for all $r=s+1, s+2, \ldots, n$ and $t=1,2, \ldots, s ;$

iii) $b_{j_{0}}=0$. Since this coefficient appears in the value of the constant $k_{r j_{0}}$ for all $r=j_{0}, j_{0}+1, \ldots, n$, they will be zero.

These results can be immediately extended to simply stable systems.

Theorem 3.5 Let $G(z)$ be a strictly proper rational simply stable transfer function. Then the following conditions are equivalent:

a) $G(z)$ admits a minimal basic bidiagonal realization with $a_{i j}>0, b_{j}>0$ and $c_{i}>0, i, j=1,2, \ldots, n$, which is simply stable.

b) $G(z)$ can be given as:

$$
G(z)=\sum_{j=1}^{n}\left(\sum_{i=j}^{n} \frac{k_{i j}}{\prod_{r=j}^{i}\left(z-a_{r}\right)}\right)
$$

where $0 \leq a_{r} \leq 1, r=1,2, \ldots, n$ and $k_{i j} \geq 0, i, j=1,2, \ldots, n$, $i \geq j$, and when $a_{i_{0}}=1$ then $k_{r t}=0$ for $r=i_{0}+1, i_{0}+2, \ldots, n$ and $t=1,2, \ldots, i_{0}$.

Proof. a) $\Rightarrow$ b) Similar to Theorem 3.3.

b) $\Rightarrow$ a) Suppose $a_{i_{0}}=1$, then

$$
\begin{aligned}
G(z)= & \frac{k_{11}}{\left(z-a_{1}\right)}+\frac{k_{21}}{\left(z-a_{2}\right)\left(z-a_{1}\right)}+\cdots+\frac{k_{i_{0} 1}}{\left(z-a_{i_{0}}\right) \cdots\left(z-a_{1}\right)}+ \\
& +\frac{k_{22}}{\left(z-a_{2}\right)}+\frac{k_{32}}{\left(z-a_{3}\right)\left(z-a_{2}\right)}+\cdots+\frac{k_{i_{0} 2}}{\left(z-a_{i_{0}}\right) \cdots\left(z-a_{2}\right)}+\cdots+ \\
& +\frac{k_{i_{0} i_{0}}}{\left(z-a_{i_{0}}\right)}+\cdots+\frac{k_{n-1, n-1}}{\left(z-a_{n-1}\right)}+\frac{k_{n, n-1}}{\left(z-a_{n}\right)\left(z-a_{n-1}\right)}+\frac{k_{n n}}{\left(z-a_{n}\right)}
\end{aligned}
$$

Following the proof of Theorem 3.3, we can obtain the positive realization $(A, B, C)$ :

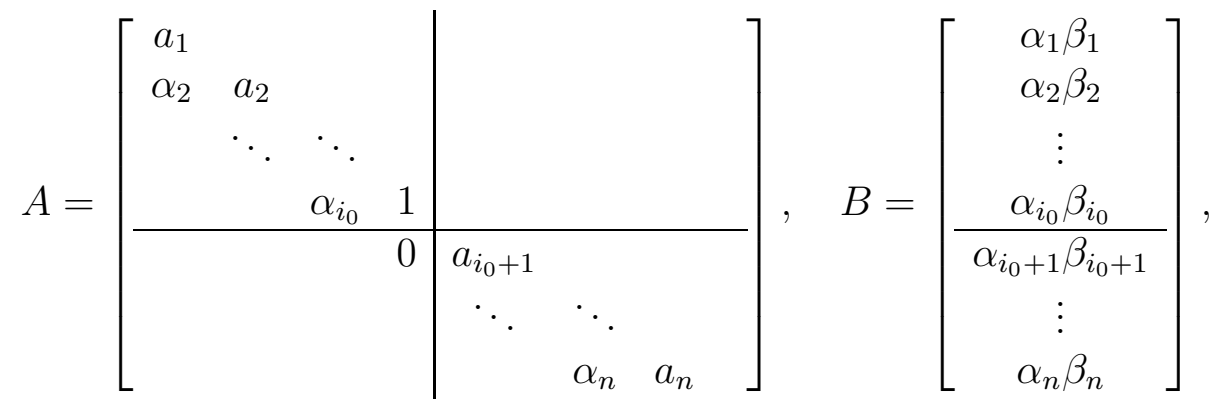


and

$$
C=\left[\begin{array}{llll|lll}
1 & 1 & \cdots & 1 & 1 & \cdots & 1
\end{array}\right]
$$

with $\alpha_{i}>0$ and $\beta_{i}>0, i=1,2, \ldots, n$. Applying Lemma 3.2 to each block of $A$, we obtain a compartmental realization that will be minimal, bidiagonal and simply stable.

Similarly, we can obtain the corresponding Corollary 3.4 for simply stable systems, i.e., Theorem 3.5 is also valid for $k_{i j} \geq 0$ taking into account the restrictions of Corollary 3.4 .

\section{$4 \quad N$-Periodic Systems}

We characterize the transfer matrix of SISO $N$-periodic compartmental systems given by a space-state model in basic bidiagonal form and even when it is triangular. To this end, we consider that an $N$-periodic system has associated $N$ invariant systems $\left(A_{s}, B_{s}, C_{s}, D_{s}\right), s=0,1, \ldots, N-1$. The space-state model of these invariant systems is explained in detail in [7]. Their transfer matrices are given by

$$
H_{s}(z)=C_{s}\left(z I-A_{s}\right)^{-1} B_{s}+D_{s}, \quad s=0,1, \ldots, N-1,
$$

which are related by the recurrence equation

$$
H_{s+1}(z)=S_{p}(z) H_{s}(z) S_{m}\left(z^{-1}\right)
$$

where the matrices $S_{p}(z)$ and $S_{m}(z)$ are defined by

$$
S_{t}(z)=\left[\begin{array}{cc}
0 & I_{(N-1) t} \\
z I_{t} & 0
\end{array}\right] .
$$

Theorem 4.1 Let an asymptotically stable SISO N-periodic compartmental system given by a space-state model in lower triangular form with $B>0$ and $C>0$. Then, the transfer matrix of this periodic system at time $s$, for $s=0,1, \ldots, N-1$, is a proper matrix

$$
H_{s}(z)=\tilde{H}_{s}(z)+D_{s}
$$

where $D_{s}$ is a real matrix and $\tilde{H}_{s}(z)$ is a strictly proper rational matrix $\tilde{H}_{s}(z)=$ $\left[H_{u v}(z)\right], u, v=1,2, \ldots, N$, whose entries are

$$
H_{u v}(z)=\sum_{j=1}^{n} \sum_{i=j}^{n} \frac{K_{i j}^{u v} P_{j}^{i-(j+1)}(z)}{\prod_{t=j}^{i}\left(z-a_{t t}\right)}
$$

with $K_{i j}^{u v}>0,0 \leq a_{t t}<1, P_{j}^{i-(j+1)}(z)$ polynomials of the degree $i-(j+1)$ and $P_{j}^{-1}=1$. 
Proof. Since the transfer matrices of the $N$ invariant systems are related by (4), we work only with the invariant system $s=0$.

$$
H_{0}(z)=C_{0}\left(z I-A_{0}\right)^{-1} B_{0}+D_{0}=\tilde{H}_{0}(z)+D_{0}
$$

where $\tilde{H}_{0}(z)$ is a strictly proper rational matrix. This matrix represents a MIMO invariant system that may be obtained by

$$
\begin{aligned}
& \tilde{H}_{0}(z)=C_{0}\left(z I-A_{0}\right)^{-1} B_{0}= \\
& =\left[\begin{array}{c}
c_{0}^{1} \\
c_{0}^{2} \\
\vdots \\
c_{0}^{N}
\end{array}\right]\left(z I-A_{0}\right)^{-1}\left[\left(b_{0}\right)_{1}\left(b_{0}\right)_{2} \cdots\left(b_{0}\right)_{N}\right]= \\
& =\left[\begin{array}{ccc}
c_{0}^{1}\left(z I-A_{0}\right)^{-1}\left(b_{0}\right)_{1} & \cdots & c_{0}^{1}\left(z I-A_{0}\right)^{-1}\left(b_{0}\right)_{N} \\
\vdots & \ddots & \vdots \\
c_{0}^{N}\left(z I-A_{0}\right)^{-1}\left(b_{0}\right)_{1} & \cdots & c_{0}^{N}\left(z I-A_{0}\right)^{-1}\left(b_{0}\right)_{N}
\end{array}\right] \\
& =\left[\begin{array}{ccc}
H_{11}(z) & \cdots & H_{1 N}(z) \\
H_{21}(z) & \cdots & H_{2 N}(z) \\
\vdots & \ddots & \vdots \\
H_{N 1}(z) & \cdots & H_{N N}(z)
\end{array}\right]
\end{aligned}
$$

Each entry $H_{u v}(z), u, v=1,2, \ldots, N$ represents a SISO system, which is usually given by:

$$
H_{u v}(z)=\sum_{i, j=1}^{n} c_{i}^{u} b_{j}^{v} \hat{a}_{i j}(z)=\sum_{i, j=1}^{n} K_{i j}^{u v} \hat{a}_{i j}(z)
$$

with

- $c_{i}^{u} \in \mathbb{R}_{+}$: component $i$ of vector $c_{0}^{u}$;

- $b_{j}^{v} \in \mathbb{R}_{+}$: component $j$ of vector $b_{0}^{v}$;

- $\hat{a}_{i j}(z)$ : component $i j$ of matrix $\left(z I-A_{0}\right)^{-1}$.

Since $A_{0}$ is a lower triangular matrix, the inverse $\left(z I-A_{0}\right)^{-1}$ is 


$$
\left(z I-A_{0}\right)^{-1}=\left[\begin{array}{cccc}
\frac{1}{\left(z-a_{11}\right)} & 0 & \cdots & 0 \\
\frac{P_{1}^{0}(z)}{\left(z-a_{22}\right)\left(z-a_{11}\right)} & \frac{1}{\left(z-a_{22}\right)} & \cdots & 0 \\
\frac{P_{1}^{1}(z)}{\left(z-a_{33}\right)\left(z-a_{22}\right)\left(z-a_{11}\right)} & \frac{P_{2}^{0}(z)}{\left(z-a_{33}\right)\left(z-a_{22}\right)} & \cdots & 0 \\
\vdots & \vdots & \ddots & \vdots \\
\frac{P_{1}^{n-2}(z)}{\left(z-a_{n n}\right)\left(z-a_{n-1, n-1}\right) \cdots\left(z-a_{11}\right)} & \frac{P_{2}^{n-3}(z)}{\left(z-a_{n n}\right) \cdots\left(z-a_{22}\right)} & \cdots & \frac{1}{\left(z-a_{n n}\right)}
\end{array}\right]
$$

where $P_{j}^{k}(z) \in \mathbb{R}[z]$ are polynomials of the degree $k$. Consequently, the entries $H_{u v}(z)$ are given by:

$$
H_{u v}(z)=\sum_{j=1}^{n}\left(\sum_{i=j}^{n} K_{i j}^{u v} \hat{a}_{i j}(z)\right)=\sum_{j=1}^{n}\left(\sum_{i=j}^{n} \frac{K_{i j}^{u v} P_{j}^{i-(j+1)}(z)}{\prod_{t=j}^{i}\left(z-a_{t t}\right)}\right)
$$

with $K_{i j}^{u v}>0,0 \leq a_{t t}<1, P_{j}^{i-(j+1)}(z)$ polynomials of the degree $i-(j+1)$ and $P_{j}^{-1}=1$.

Remark 4.2 We have proved that these polynomials $P_{j}^{i-(j+1)}(z)$ have positive coefficients for $N$-periodic systems with $n \leq 4$ and $N \leq 4$ given by a space-state model in basic bidiagonal form. Tedious computation leads us to suppose that this result follows for arbitraries $n$ and $N$.

Note that we have considered the case of compartmental systems with compartments connected in series, but what happens when some compartments are connected in parallel? Using a similar proof of Theorem 4.1, we can deduce the following result.

Corollary 4.3 If the asymptotically stable SISO compartmental system has $n$ compartments connected in parallel, then the entries of the strictly proper rational matrix $H_{u v}(z)$ are given by:

$$
H_{u v}(z)=\sum_{i=1}^{n} \frac{K_{i i}^{u v}}{\left(z-a_{i i}\right)}
$$

with $K_{i i}^{u v}>0,0 \leq a_{i i}<1$.

Expression (6) has fewer terms than (5) and polynomials $P_{j}^{i-(j+1)}(z)$ of the numerator are 1 for all $i, j=1,2, \ldots, n$. If the system has some compartments connected in parallel and others in series, then the strictly proper rational matrix is a combination of the two expressions given in (5) and (6). 
Corollary 4.4 If the asymptotically stable SISO compartmental system has the first $r$ compartments in series, the following s compartments in parallel and the last $n-r-s$ compartments in series again, then the entries of the strictly proper rational matrix $H_{u v}(z)$ are given by:

$$
\begin{aligned}
H_{u v}(z) & =\sum_{j=1}^{r}\left(\sum_{i=j}^{r} \frac{K_{i j}^{u v} P_{j}^{i-(j+1)}(z)}{\prod_{t=j}^{i}\left(z-a_{t t}\right)}\right)+\sum_{t=r+1}^{r+s} \frac{K_{i j}^{u v}}{\left(z-a_{t t}\right)}+ \\
& +\sum_{\substack{j=\\
r+s+1}}^{n}\left(\sum_{i=j}^{n} \frac{K_{i j}^{u v} P_{j}^{i-(j+1)}(z)}{\prod_{t=j}^{i}\left(z-a_{t t}\right)}\right)
\end{aligned}
$$

with $K_{i j}^{u v}>0,0 \leq a_{t t}<1, P_{j}^{-1}=1$ and $P_{j}^{i-(j+1)}(z)$ polynomials with positive coefficients of the degree $i-(j+1)$.

Finally, it is worth noting that when the system has compartments without input, output or neither of the two, the number of terms in the expression of the entries $H_{u v}(z)$ also changes.

ACKNOWLEDGEMENTS. This work has been supported by the Spanish DGI and FEDER grant AGL2004-03263/AGR and by the UPV under its research program.

\section{References}

[1] A. Astolfi and P. Colaneri, A note on existence of positive realizations, Linear Algebra and its Applications, 390 (2004), 329 - 343.

[2] L. Benvenuti and L. Farina, Positive and Compartmental Systems, IEEE Transactions on Automatic Control, 47 (2002), 370 - 373.

[3] L. Benvenuti, L. Farina, B.D.O. Anderson and F. De Bruyne, Minimal Positive Realizations of Transfer Functions with Positive Real Poles, IEEE Transactions on Circuits and Systems I: Fundamental Theory and Applications, 47 (2000), 1370 - 1377.

[4] A. Berman and R.J. Plemmons, Nonnegative Matrices in the Mathematical Sciences, SIAM, Philadelphia, 1994.

[5] G.I. Bischi, Compartmental analysis of economic systems with heterogeneous agents: an introduction, Beyond the Representative Agent, A. Kirman and M. Gallegati Eds. Elgar Publ. Co. (1998), 181 - 214. 
[6] R. Bru, C. Coll, V. Hernández and E. Sánchez, Geometrical Conditions for the Reachability and Realizability of Positive Periodic Discrete Systems, Linear Algebra and its Applications, 256 (1997), 109 - 124.

[7] C. Coll, R. Bru, E. Sánchez and V. Hernández, Discrete-Time Linear Periodic Realization in the Frequency Domain, Linear Algebra and its Applications, 203-204 (1994), 301 - 326.

[8] L. Farina and S. Rinaldi, Positive Linear Systems. Theory and Applications, Pure and Applied Mathematics, Wiley, New York, 2000.

[9] J.A. Jacquez and C.P. Simon, Qualitative theory of compartmental systems, SIAM Review, 35 (1993), 43 - 79.

[10] T. Kaczorek, Positive 1D and 2D Systems, Springer, London, 2002.

[11] H. Maeda and S. Kodama, Positive Realizations of Difference Equations, IEEE Transactions on Circuits ans Systems, 28 (1981), 39 - 47.

[12] H. Maeda, S. Kodama and F. Kajiya, Compartmental System Analysis: Realization of a Class of Linear Systems with Physical Constraints, IEEE Transactions on Circuits and Systems, 22 (1977), 8 - 14.

[13] Y. Ohta, H. Maeda and S. Kodama, Reachability, observability and realizability of continuous-time positive systems, SIAM J. Control Optim., 22 (1984), 171 - 180.

[14] J.M. Van den Hof, Structural identifiability of linear compartmental systems, IEEE Transactions on Automatic Control, 43 (1998), 800 - 818.

\section{Received: June 27, 2006}

